\title{
Phantom of the Universe: A Planetarium Show about Dark Matter
}

\author{
R. Michael Barnett ${ }^{1}$ \\ Lawrence Berkeley National Laboratory \\ Mailstop 50R-6008 \\ 1 Cyclotron Road \\ Berkeley, CA 94720 USA \\ E-mail: rmbarnett@1bl.gov
}

\section{Kaushik De}

University of Texas, Arlington

Department of Physics, Box 19059, Arlington, Texas 76019, USA

E-mail: kaushik@uta.edu

\section{Reinhard Schwienhorst}

Michigan State University

Department of Physics and Astronomy

3241 Biomedical and Physical Sciences

567 Wilson Road

East Lansing, MI 48824-2320, USA

E-mail: schwier@pa.msu.edu

\begin{abstract}
A new planetarium show about Dark Matter is entitled "Phantom of the Universe". Recently completed, it features the exciting story of dark matter, from galaxies to a deep underground experiment to the Large Hadron Collider. It is being distributed for free to planetariums worldwide.
\end{abstract}

38th International Conference on High Energy Physics 3-10 August 2016

Chicago, USA

\footnotetext{
${ }^{1}$ Speaker

(c) Copyright owned by the author(s) under the terms of the Creative Commons

Attribution-NonCommercial-NoDerivatives 4.0 International License (CC BY-NC-ND 4.0).
} 


POS $\quad \begin{aligned} & \text { PROCEEDINGS } \\ & \text { OF SCIENCE }\end{aligned}$

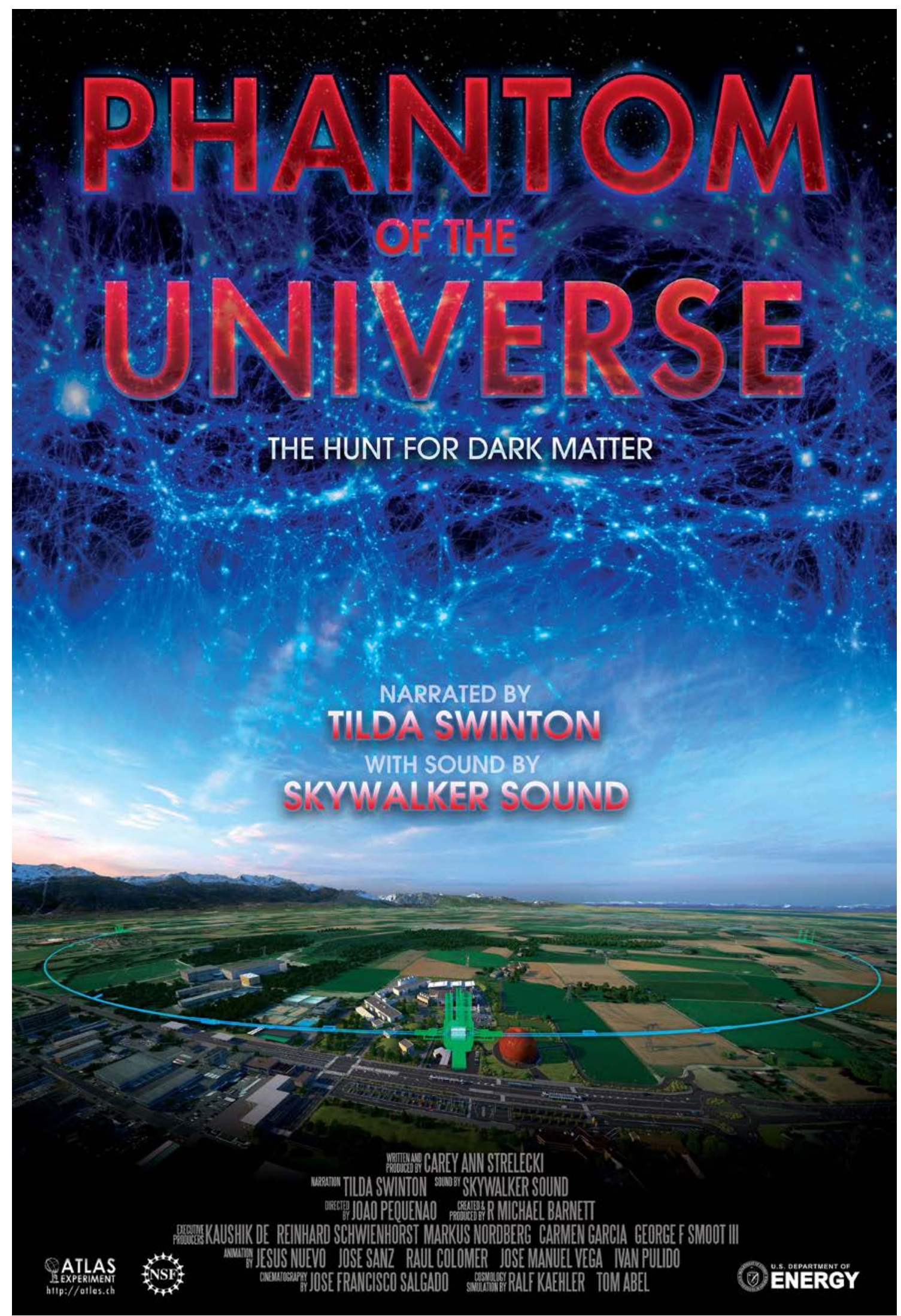




\section{Introduction}

Around the world, the search for a phantom continues. A new planetarium show is previewing the discovery of this phantom - dark matter - in astrophysics, at the Sanford Underground Research Facility, as well as in astrophysics and at the Large Hadron Collider (LHC).

This new show stars Academy Award winner Tilda Swinton as narrator, with sound effects from an Academy Award winning team at Skywalker Sound, and guidance from Nobel Laureate George Smoot. The director is head of the Media Lab at CERN.

The title "Phantom of the Universe" reflects dark matter's presence everywhere in the universe from galaxies to deep underground.

Three years in the making, the show is being distributed for free to planetariums worldwide. It is expected to premiere in several cities including London (date to be determined) and already premiered in Vienna starting 18 October 2016.

See: http://PhantomOfTheUniverse.org

\subsection{Production Team for the Planetarium Show}

The show's creators and executive producers are Michael Barnett, Lawrence Berkeley National Lab, Kaushik De of the University of Texas, Arlington, Reinhard Schwienhorst of Michigan State University, Carmen Garcia of the University of Valencia, Markus Nordberg of CERN and George Smoot of the University of California, Berkeley.

With music composed by Skywalker Sound (Lucas Films ) and narration by acclaimed Academy-Award winning actress, Tilda Swinton, Phantom of the Universe showcases the creativity and directing prowess of Joao Pequenao of CERN's Media Lab and the writing and producing talents of award-winning filmmaker, Carey Ann Strelecki, PGA, WGA.

A talented team based at the Instituto de Física Corpuscular (IFIC) de la Universitat de València produced the animated sequences of the show. Animation Technical Director Jesus Nuevo, with colleagues Jose Sanz, Raul Colomer, Jose Manuel Vega and Ivan Pulido gave life to the visuals.

The dynamic live-action scenes were filmed by Jose Francisco Salgado of the Adler Planetarium. Some of the cosmology simulations were produced by Ralf Kaehler and Tom Abel of KIPAC at the SLAC lab.

\section{Content of the Show}

The show reveals the first hints of dark matter's existence through the eyes of Fritz Zwicky, the scientist who coined the term "dark matter." It describes the astral choreography witnessed by Vera Rubin in the Andromeda galaxy.

We learn how scientists around the world are collaborating to track down the constituents of dark matter. It plummets deep underground to see the LUX dark matter detector. The production includes camera footage taken at the SURF, including a ride on a rail car through a 4,850-foot-deep tunnel.

From there, it journeys across space to the LHC at CERN, speeding alongside particles before they collide in visually stunning explosions of light and sound. 
An obvious challenge for this production, "Phantom of the Universe," is that the star of the show-dark matter, which we have so far detected only through its gravitational effects though it makes up an estimated 85 percent of the total mass of the universe-is invisible and we don't yet know what it's made of. Researchers are scanning the night sky and designing ultrasensitive particle detectors in hopes of solving its mysteries.

The show conveys the excitement of what's going on in research, how many things we've learned about dark matter, how we know it's really there, and to show how we're trying to figure out what it is.

\section{Planetarium Shows and Audiences}

Besides the ability to display imagery above and all around you, planetariums are also a powerful outreach and educational platform, drawing about 120 million visitors each year. "Phantom of the Universe" is being offered free of charge to planetariums around the world and has the potential to reach millions of viewers.

The 25-minute production was recently completed. A German-language version was shown October 18 during the opening of a new exhibit about particle physics and cosmology in Vienna, and a French-language version is in progress. Details of special advance screenings in the U.S. will be announced soon.

We built huge and complex scenes. The rendering process is very intensive and slow, so you need to have a lot of computers available to distribute these calculations.

Modern planetariums have up to six projectors that work together to display a show.

\section{Behind the Scenes}

The scientific process and filmmaking are both creative processes, and connecting art to science is what we're trying to do with this film. Our purpose here is to get people excited about physics and cosmology.

A highlight was the opportunity to meet and work with actress Tilda Swinton, In June, Barnett traveled to a small recording studio near the North Sea in the Scottish Highlands to witness Swinton recording the narration.

Our moment of truth is when we go to a screening (such as in Vienna), and we see adults and kids excited about understanding dark matter.

\section{Support}

The collaboration that created "Phantom of the Universe" included Berkeley Lab; University of Texas in Arlington; Michigan State University, and the Instituto de Física Corpuscular (IFIC), a joint center of the Spanish National Research Council (CSIC) and València University.

The production was supported by:

Department of Energy's Office of High Energy Physics

The National Science Foundation

ATLAS Experiment

Lawrence Berkeley National Laboratory

Michigan State University

University of Texas at Arlington

CERN (Geneva, Switzerland) 
Science \& Technology Facilities Council (UK)

Instituto de Física Corpuscular, IFIC (Spain)

Institut für Hochenergiephysik der Österreichischen Akademie der Wissenschaften

Naturhistorisches Museum Wien

DESY, Deutsches Elektronen-Synchrotron (Germany)

\section{Website for Phantom of the Universe}

Find clips from the show (in circle format) and the latest updates at: http://PhantomOfTheUniverse.org

\section{Acknowledgments}

We have benefitted from and enjoyed the pleasure of working with many professionals from the worlds of particle physics, cosmology, filmmaking, animation, planetariums, and astrophysical simulations. These are truly amazing people. We particularly wish to thank Joao Pequenao, Carey Ann Strelecki, and our team of animators led by Jesus Nuevo. 